May 2002

\title{
Ordinary atom-mirror atom bound states: A new window on the mirror world
}

\author{
R. Foot ${ }^{a}$ and S. Mitra ${ }^{b}$ \\ afoot@physics.unimelb.edu.au \\ School of Physics \\ Research Centre for High Energy Physics \\ The University of Melbourne \\ Victoria 3010 Australia \\ bsaibalm@science.uva.nl \\ Instituut voor Theoretische Fysica \\ Universiteit van Amsterdam \\ 1018 XE Amsterdam \\ The Netherlands
}

\begin{abstract}
Mirror symmetry is a plausible candidate for a fundamental symmetry of particle interactions which can be exactly conserved if a set of mirror particles exist. The properties of the mirror particles seem to provide an excellent candidate to explain the inferred dark matter of the Universe and might also be responsible for a variety of other puzzles in particle physics, astrophysics, meteoritics and planetary science. One such puzzle - the orthopositronium lifetime problem - can be explained if there is a small kinetic mixing of ordinary and mirror photons. We show that this kinetic mixing implies the existence of ordinary atom - mirror atom bound states with interesting terrestrial and astrophysical implications. We suggest that sensitive mass spectroscopic studies of ordinary samples containing heavy elements such as lead might reveal the presence of these bound states, as they would appear as anomalously heavy elements. In addition to the effects of single mirror atoms, collective effects from embedded fragments of mirror matter (such as mirror iron microparticles) are also possible. We speculate that such mirror matter fragments might explain a mysterious UV photon burst observed coming from a laser irradiated lead target in a recent experiment.
\end{abstract}


One of the most natural candidates for a symmetry of nature is parity symmetry (also called left-right or mirror symmetry). While it is an established experimental fact that parity symmetry appears broken by the interactions of the known elementary particles, this however does not exclude the possible existence of exact unbroken parity symmetry in nature. This is because parity (and also time reversal) can be exactly conserved if a set of mirror particles exist [1, 2]. The idea is that for each ordinary particle, such as the photon, electron, proton and neutron, there is a corresponding mirror particle, of exactly the same mass as the ordinary particle ?. Furthermore, the mirror particles interact with each other in exactly the same way that the ordinary particles do. It follows that the mirror proton is stable for the same reason that the ordinary proton is stable, and that is, the interactions of the mirror particles conserve a mirror baryon number. The mirror particles are not produced (significantly) in laboratory experiments just because they couple very weakly to the ordinary particles. In the modern language of gauge theories, the mirror particles are all singlets under the standard $G \equiv S U(3) \otimes S U(2)_{L} \otimes U(1)_{Y}$ gauge interactions. Instead the mirror fermions interact with a set of mirror gauge particles, so that the gauge symmetry of the theory is doubled, i.e. $G \otimes G$ (the ordinary particles are, of course, singlets under the mirror gauge symmetry)[2]. Parity is conserved because the mirror fermions experience $V+A$ (right-handed) mirror weak interactions and the ordinary fermions experience the usual $V-A$ (lefthanded) weak interactions. Ordinary and mirror particles interact with each other predominately by gravity only.

At the present time there is a fascinating range of experimental observations supporting the existence of mirror matter, for a review see Ref. [5] (for a more detailed discussion of the case for mirror matter, accessible to the non-specialist, see the recent book [6]). The evidence includes numerous observations suggesting the existence of invisible 'dark matter' in galaxies. Mirror matter is stable and dark and provides a natural candidate for this inferred dark matter [7]. The MACHO observations [8, close-in extrasolar planets 9], isolated planets [10 and even gamma ray bursts 11 may all be mirror world manifestations. In our solar system, the anomalous slowing down of the Pioneer spacecraft 12, the Tunguska event and some anomalous low altitude fireball phenomena 13, 14 have also been identified as possible manifestations of the mirror world. On the quantum level, small fundamental interactions connecting ordinary and mirror matter are possible. Theoretical constraints from gauge invariance, renormalizability and mirror symmetry suggest only three possible types of interactions 2, 15]: photonmirror photon kinetic mixing, neutrino-mirror neutrino mass mixing and Higgsmirror Higgs interactions. The main experimental implication of photon-mirror photon kinetic mixing is that it modifies the properties of orthopositronium, leading to a shorter effective lifetime in 'vacuum' experiments 16, 17, 18]. A shorter lifetime is in fact seen at the 5 sigma level! 19, 18. Neutrino-mirror neutrino mass mixing implies maximal oscillations for each ordinary neutrino with its mirror partner - a result which may be connected with the neutrino physics anomalies 15,20 .

\footnotetext{
1 The mirror particles only have the same mass as their ordinary counterparts provided that the mirror symmetry is unbroken. It is possible to write down gauge models where the mirror symmetry is broken[3, in some cases allowing the mirror particles to have completely arbitrary masses [], however these scenarios tend to be more complicated and much less well motivated in our view.
} 
Of the few possible ways in which ordinary and mirror matter can interact with each other besides gravity, of most importance for this paper is the photonmirror photon kinetic mixing interaction. In field theory, photon-mirror photon kinetic mixing is described by the interaction

$$
\mathcal{L}=\frac{\epsilon}{2} F^{\mu \nu} F_{\mu \nu}^{\prime}
$$

where $F^{\mu \nu}\left(F_{\mu \nu}^{\prime}\right)$ is the field strength tensor for electromagnetism (mirror electromagnetism). This type of Lagrangian term is gauge invariant and renormalizable and can exist at tree level[2, 21] or may be induced radiatively in models without $U(1)$ gauge symmetries (such as grand unified theories) 16, 22, 23. One effect of ordinary photon-mirror photon kinetic mixing is to give the mirror charged particles a small electric charge 2, 16, 22. That is, they couple to ordinary photons with electric charge $\epsilon e$.

The most important experimental implication of photon-mirror photon kinetic mixing is that it modifies the properties of orthopositronium 16. This effect arises due to radiative off-diagonal contributions to the orthopositronium, mirror orthopositronium mass matrix. This means that orthopositronium oscillates into its mirror partner. Decays of mirror orthopositronium are not detected experimentally which effectively increases the observed decay rate 16]. Because collisions of orthopositronium destroy the quantum coherence, this mirror world effect is most important for experiments which are designed such that the collision rate of the orthopositronium is low 17. The only accurate experiment sensitive to the mirror world effect is the Ann Arbour vacuum cavity experiment 19]. This experiment obtained a decay rate of $\Gamma_{o P s}=7.0482 \pm 0.0016 \mu \mathrm{s}^{-1}$. Normalizing this measured value with the current theoretical value of $7.0399 \mu \mathrm{s}^{-1}$ [24] gives

$$
\frac{\Gamma_{o P s}(\text { exp })}{\Gamma_{o P s}(\text { theory })}=1.0012 \pm 0.00023
$$

which is a five sigma discrepancy with theory. It suggests a value $|\epsilon| \simeq 10^{-6}$ for the photon-mirror photon kinetic mixing 18]. Taken at face value this experiment is strong evidence for the existence of mirror matter and hence parity symmetry. The sign of $\epsilon$ is not constrained by the orthopositronium experiments and so there are two distinct possibilities depending on whether $\epsilon>0$ or $\epsilon<0$.

Perhaps one of the most fascinating implications of the photon - mirror photon kinetic mixing interaction occurs if there are small mirror matter bodies in our solar system. It has been suggested in Ref. $13,5,14$, 14 the collisions of such bodies with the Earth may naturally explain many puzzling features of the 1908 Tunguska event as well as the low altitude anomalous fireball events. An important implication of this explanation is that mirror matter fragments should exist in the ground at these impact sites. Clearly, an important issue is how to search for mirror matter in the ground. One concrete idea was proposed in Ref.[14], where it was suggested that mirror matter in the ground can cool the surrounding ordinary matter by drawing in heat and radiating it away as mirror photons. In this paper we will discuss a complementary way of searching for mirror matter in the ground.

\footnotetext{
${ }^{2}$ Actually, the cooling effect of mirror matter that is vapourized in the atmosphere (from e.g. small mirror matter space-bodies or dust particles colliding with the Earth) might also lead to interesting effects. For example, one might think that rapid cooling of the atmosphere
} 
While the force of gravity can be opposed by electrostatic forces for a solid piece of mirror matter (if $|\epsilon| \sim 10^{-6}$ ), one might expect mirror matter in gaseous form to eventually diffuse to the center of the Earth. This is only true, though, if the mirror atoms cannot bind with ordinary atoms at ordinary temperatures. If the effective ordinary electric charge of the mirror nuclei is of opposite sign to ordinary nuclei (i.e. $\epsilon<0$ ), then ordinary - mirror nuclei can form bound states. Consider the case of a proton and a mirror proton. In this case, the Bohr radius of the proton - mirror proton bound state $\left(R_{b}\right)$ and its binding energy $\left(E_{b}\right)$ are given by (we use natural units where $\hbar=c=1$ throughout):

$$
\begin{aligned}
& R_{b}=\frac{m_{e}}{\mu \epsilon} r_{b o h r}^{H}=\frac{1}{\mu|\epsilon| e^{2}} \sim 10^{3}\left(\frac{10^{-6}}{|\epsilon|}\right) r_{b o h r}^{H}, \\
& E_{b}=\frac{\epsilon^{2} \mu}{m_{e}} E_{b o h r}^{H}=\frac{-\mu \epsilon^{2} e^{4}}{2} \sim-10^{-8}\left(\frac{\epsilon}{10^{-6}}\right)^{2} \mathrm{eV},
\end{aligned}
$$

where $\mu=m_{p} / 2$ is the reduced mass and $r_{b o h r}^{H}, E_{b o h r}^{H}$ are the standard Bohr radius and ground state energy for Hydrogen. Clearly, the binding energy is too low to be of much interest if $|\epsilon| \approx 10^{-6}$ as suggested by the orthopositronium experiments. However, the binding energy increases for larger masses and also for larger charges.

Perhaps an interesting observation is that if the Bohr radius of the ordinary - mirror nuclei bound state is less than the Bohr radius of the inner electrons and mirror electrons, then the electric charge of the ordinary nuclei and mirror nuclei are, to a good approximation, not screened by the electrons and mirror electrons. This means that we can, to a good approximation, ignore the electrons and mirror electrons and treat atoms as nuclei. The condition for this to occur is that:

$$
\begin{aligned}
& R_{b} \lesssim r_{b}^{K} \Rightarrow \frac{1}{\mu|\epsilon| Z_{1} Z_{2} e^{2}} \lesssim \frac{1}{m_{e} e^{2} Z_{1}} \\
& \text { That is, } \quad Z_{2} \gtrsim \frac{m_{e}}{\mu} \frac{1}{|\epsilon|},
\end{aligned}
$$

where we have assumed $Z_{1} \geq Z_{2}$ and used the notation $r_{b}^{K}$ for the Bohr radius of the inner K-shell electrons. For light elements, $Z_{1}, Z_{2}<15$, the above condition is never satisfied; which means that ordinary - mirror atom light element bound states do not exist or have extremely low binding energies (certainly too low to exist on Earth). However, for heavy elements, $Z_{1}, Z_{2} \gtrsim 15$, the above condition is satisfied. Taking as an example, $F e-F e^{\prime}$ bound state, then $R_{b}=0.7 r_{b}^{K}$, verifying that the ordinary and mirror atom are bound so closely that the screening effects of the ordinary and mirror electrons can be approximately ignored. The binding energy of the ground state in the $F e-F e^{\prime}$ system is approximately:

$$
\begin{aligned}
E_{F e-F e^{\prime}} & \simeq \frac{-\epsilon^{2} m_{F e} Z_{F e}^{4} e^{4}}{4} \\
& \simeq 0.3\left(\frac{\epsilon}{10^{-6}}\right)^{2} \mathrm{eV} .
\end{aligned}
$$

due to mirror matter absorbing heat from the surrounding ordinary atoms (and radiating that heat way as mirror photons) might lead to the formation of clouds and maybe even ice blocks. Perhaps this might be connected with long standing observations of atmospheric holes 25] and observations of falling ice blocks 26. 
While this particular bound state could have interesting implications in cold environments such as the ISM, it would not exist indefinitely at ordinary temperatures for single $F e^{\prime}$ atoms. They would occasionally be excited by thermal interactions, eventually diffusing towards the Earth's center. However, heavier bound states have higher binding energies and can be quite stable at ordinary temperatures. For example, the binding energy of $\mathrm{Pb}-F e^{\prime}$ is about $5 \mathrm{eV}$ and $P b-P b^{\prime}$ is about $120 \mathrm{eV}$.

The above observations open several new ways to search for mirror matter. For example, if mirror iron meteorites exist, then such bodies can vaporise in the atmosphere. Each iron atom would diffuse towards the ground, where it would only be stopped (permanently) if it encountered an ordinary element heavier than $F e$, e.g. lead (or it encounted some embedded mirror matter fragment). Thus, ordinary samples of lead, may contain a small fraction of $P b-F e^{\prime}$ bound states which have built up over time. [Mirror iron may also come from cosmic rays, since we know that ordinary cosmic rays contain $F e$, it is very plausible that they might also contain $F e^{\prime}$.]

The capture cross section of say, $F e^{\prime}$ on lead can be estimated to be 3 :

$$
\sigma_{r c}=\frac{256 \pi^{2}}{3 m_{F e}^{2}} Z_{F e}^{2} \alpha \frac{\xi^{6}}{\left(1+\xi^{2}\right)^{4}} \frac{\exp (2 \xi \tau)}{1-\exp (-2 \pi \xi)}
$$

where $\sin \tau=\frac{-2 \xi}{1+\xi^{2}}$ and $\xi=\epsilon \alpha Z_{F e} Z_{P b} / v$. In order to work out $\sigma_{r c}$ for the capture of $F e^{\prime}$ on lead, we need to first estimate the velocity, $v$, of the $F e^{\prime}$ atoms. For the interesting case of $|\epsilon| \approx 10^{-6}$, the $F e^{\prime}$ undergoes many elastic collisions with the ordinary nuclei so that by the time it could reach any lead deposit in the ground, an individual $F e^{\prime}$ atom would have thermalized with the surroundings. This means that the mean energy of an individual $F e^{\prime}$ atom would be approximately,

$$
\begin{aligned}
& \langle E\rangle \quad \approx \frac{3}{2} k T, \\
& \Rightarrow \quad v / c \approx \sqrt{\frac{3 k T}{m_{F e}}} \approx 10^{-6} .
\end{aligned}
$$

If $|\epsilon| \approx 10^{-6}$ as suggested by the orthopositronium experiments then $\xi \approx 13$ and $\sigma_{r c}$ is

$$
\sigma_{r c} \approx 3 \times 10^{-30} \mathrm{~cm}^{2}
$$

This means that $F e^{\prime}$ must travel an average total length of $\ell=1 / n_{\text {Lead }} \sigma_{r c} \approx$ $3 \times 10^{7} \mathrm{~km}$ before being captured by lead (where we have used the mean number density of lead atoms in the Earth's crust of $n_{\text {lead }} \sim 10^{17} / \mathrm{cm}^{3}$ ). The average time needed for $F e^{\prime}$ to be captured by lead is thus about $\ell / v \approx 3$ years. Because the elastic scattering with nuclei is much larger $\left(\sigma \sim 10^{-20} \mathrm{~cm}^{2}\right), F e^{\prime}$ will be captured by lead at a distance much smaller than $\ell$. An $F e^{\prime}$ atom has a mean free path in the earth's crust of about $\ell_{m}=1 / n \sigma \sim 10^{-3} \mathrm{~cm}$. The $F e^{\prime}$ atom will thus typically perform a random walk of about $N=\ell / \ell_{m} \sim 3 \times 10^{15}$ steps

\footnotetext{
3 This cross section can be obtained from the standard formula for the capture of a charge electron on a nucleus (see e.g. Ref.[27]), after the replacements $e \rightarrow Z_{F e} e, Z_{\text {nucleus }} \rightarrow \epsilon Z_{P b}$ and $m_{e} \rightarrow m_{F e}$.
} 
of length $\ell_{m}$. The typical distance a $F e^{\prime}$ atom will stray before being captured by lead is thus about $\sqrt{N} \times \ell_{m} \approx 500$ meters.

It is also possible for $F e^{\prime}$ (and other potential heavy mirror elements) to be absorbed by colliding with any mirror matter fragments that happen to be embedded in the Earth. The strength of the capture process relative to condensation on mirror matter fragments is of course difficult to quantify. For this reason and because the (time) integrated flux of $F e^{\prime}$ atoms striking the Earth (from e.g. the vapourization of $F e^{\prime}$ meteorites in the atmosphere or from cosmic rays if they contain an $F e^{\prime}$ component) is essentially unknown, it is impossible to give precise predictions for the exotic ordinary - mirror atom bound state abundances in materials containing heavy elements such as lead.

Interestingly, a recent experiment has searched for bound states consisting of strongly interacting massive particles (SIMPS) and iron and gold nuclei [29]. No such bound states were found. Using these results an upper limit of about $10^{-11}$ can be set for $A u-F e^{\prime}$ bound states abundances, and an upper limit of $10^{-10}$ for $A u-P b^{\prime}$ bound states abundances. Samples near the Earth's surface were tested, because SIMPS (being strongly interacting) are assumed to have a penetration depth of a few meters or less. Since a mirror atom is expected to penetrate much deeper into the Earth before being captured, it is not necessary to focus on samples near the Earth's surface. Any sample containing heavy elements such as lead could be used. In particular, a much more senstive search for ordinary-mirror atom bound states could be preformed by working with liquid compounds such as lead tetra chloride $\left(\mathrm{PbCl}_{4}\right)$. Such liquids could be put into a centrifuge, thereby greatly enhancing the concentration of, say, $\mathrm{Pb}-$ $\mathrm{Fe}^{\prime}-\mathrm{Cl}_{4}$ (in this example). After this purification procedure the sample could be then put in a mass spectrometer. In this way a very sensitive limit on the abundance of such exotic states could be achieved (perhaps a sensitivity of order $10^{-16}$ could be expected).

While our discussion has focused on the capture of single $F e^{\prime}$ atoms, interesting effects can also occur for $F e^{\prime}$ fragments (and other heavy mirror elements), including tiny micron-sized mirror iron particles containing e.g. $\sim 10^{8} \mathrm{Fe}^{\prime}$ atoms. Such fragments would be stopped in the Earth after only a very short distance (typically less than a millimeter). Thus, unlike the case of individual atoms, small fragments and microparticles might be expected to occur only in lead samples exposed to the atmosphere. The binding energy of the fragment depends on several factors including its crystal structure and lattice spacing. At ordinary temperatures lead forms a face-centered cubic structure while iron forms a body-centered cubic structure. Depending on how these lattices are arranged relative to each other, there may be several possible stable configurations. The state that an embedded fragment happens to be in might not be the lowest energy configuration. This point is illustrated in Figure 1, where the system is in the second local minimum. In this case it might be possible to cause a phase transition to a lower energy configuration by irradiating the sample with a laser resulting in emission of UV photons with higher energy than the laser photons if the final configuration involves $P b-F e^{\prime}$ bound states. Interestingly, there is a recent experiment which has indeed found such a puzzling burst of UV photons from laser irradiated lead targets 28]. Further work needs to be done, both experimentally and theoretically to see if this mirror matter explanation could really be the origin of those observations.

In addition to terrestrial implications, one might also imagine interesting 
astrophysical implications of ordinary-mirror mixed matter. If, for example, the interstellar medium (ISM) has dust particles containing both ordinary and mirror fragments. The interstellar medium is a very cold environment which means that even relatively light states such as $F e-F e^{\prime}$ or $F e-M g^{\prime}$ could exist leading to infra-red emission lines. Also heavier states such as $P b-F e^{\prime}$ (or $P b^{\prime}-F e$ ) could also exist but would have absorption in the UV. Interestingly there are many unidentified infra-red, optical and UV lines in the ISM (for example the well known UV absorption feature at $\lambda=2175 A$ ), but we postpone a more detailed analysis for the future.

In conclusion, we have pointed out that the small photon - mirror photon kinetic mixing interaction - suggested by orthopositronium experiments - should cause single heavy mirror atoms (such as $F e^{\prime}$ ) to become bound with heavy ordinary atoms. These bound states could be found if sensitive mass spectroscopy was done on materials such as ordinary lead. The existence of these bound states could also lead to interesting implications if small mirror fragments (such as iron microparticles) are stopped in ordinary materials containing heavy elements such as lead. We have speculated that this might explain a recent laser experiment which has found an unexpected burst of UV light from laser irradiated lead targets. The ordinary - mirror atom bound states may also have interesting astrophysical implications if such mixed matter exists in the ISM.

\section{Acknowledgements}

We would like to thank A. Pakhomov for useful correspondence regarding his experiment. R.F. wishes to acknowledge very useful and interesting discussions/correspondence with J. Learned, J. Martinez-Frias, S. Pakvasa, R. Volkas, T. L. Yoon and wishes to thank T. Mesirow for bringing the laser experiment to his attention.

\section{References}

[1] T. D. Lee and C. N. Yang, Phys. Rev. 104, 256 (1956); I. Kobzarev, L. Okun and I. Pomeranchuk, Sov. J. Nucl. Phys. 3, 837 (1966); M. Pavsic, Int. J. Theor. Phys. 9, 229 (1974).

[2] R. Foot, H. Lew and R. R. Volkas, Phys. Lett. B272, 67 (1991).

[3] R. Foot and H. Lew, hep-ph/9411390, July 1994; R. Foot, H. Lew and R. R. Volkas, JHEP 0007, 032 (2000).

[4] Z. Berezhiani and R. N. Mohapatra, Phys. Rev. D52, 6607 (1995); Z. Berezhiani, A. Dolgov and R. Mohapatra, Phys. Lett. B375, 26 (1996); R. N. Mohapatra and V. L. Teplitz, Phys. Lett. B462, 302 (1999).

[5] R. Foot, Acta Phys. Polon. B32, 2253 (2001).

[6] Shadowlands: Quest for Mirror Matter in the Universe, Universal Publishers, 2002, by R. Foot. 
[7] S. I. Blinnikov and M. Yu. Khlopov, Sov. J. Nucl. Phys. 36, 472 (1982); Sov. Astron. 27, 371 (1983); E. W. Kolb, M. Seckel and M. S. Turner, Nature 514, 415 (1985); M. Yu. Khlopov et al, Soviet Astronomy, 35, 21 (1991); M. Hodges, Phys. Rev. D47, 456 (1993); N. F. Bell and R. R. Volkas, Phys. Rev. D59, 107301 (1999); S. Blinnikov, Surveys High Energy Physics, 15, 37 (2000); V. Berezinsky and A. Vilenkin, Phys. Rev. D62, 083512 (2000); Z. Berezhiani, D. Comelli and F. L. Villante, Phys. Lett. B503, 362 (2001).

[8] Z. K. Silagadze, Phys. At. Nucl. 60, 272 (1997); S. Blinnikov, astroph/9801015; R. Foot, Phys. Lett. B452, 83 (1999).

[9] R. Foot, Phys. Lett. B471, 191 (2000); Phys. Lett. B505, 1 (2001).

[10] R. Foot, A. Yu. Ignatiev and R. R. Volkas, astro-ph/0010502 (to appear in Astroparticle Physics).

[11] S. Blinnikov, astro-ph/9902305.

[12] R. Foot and R. R. Volkas, Phys. Lett. B517, 13 (2001).

[13] R. Foot, Acta Phys. Polon. B32, 3133 (2001).

[14] R. Foot and T. L. Yoon, astro-ph/0203152.

[15] R. Foot, H. Lew and R. R. Volkas, Mod. Phys. Lett. A7, 2567 (1992).

[16] S. L. Glashow, Phys. Lett. B167, 35 (1986).

[17] S. N. Gninenko, Phys. Lett. B326, 317 (1994).

[18] R. Foot and S. N. Gninenko, Phys. Lett. B480, 171 (2000).

[19] J. S. Nico, D. W. Gidley, A. Rich and P. W. Zitzewitz, Phys. Rev. Lett. $65,1344(1990)$.

[20] R. Foot, Mod. Phys. Lett. A9, 169 (1994); R. Foot and R. R. Volkas, Phys. Rev. D52, 6595 (1995).

[21] R. Foot and X-G. He, Phys. Lett. B267, 509 (1991).

[22] B. Holdom, Phys. Lett. B166, 196 (1986).

[23] M. Collie and R. Foot, Phys. Lett. B432, 134 (1998).

[24] G. S. Adkins, R. N. Fell and J. Sapirstein, Phys. Rev. Lett. 84, 5086 (2000).

[25] http://smallcomets.physics.uiowa.edu

[26] http://tierra.rediris.es/bloquesdehield and J. Martinez-Frias et al, Compositional Heterogeneity of Hailstones: Atmospheric Conditions and Possible Environmental Implications, Ambio Vol. 30 (2001). See also http://www.geocities.com/olkhov/gr1997.htm where a catalogue of many strange atmospheric events (of unknown origin) is given.

[27] See e.g. V. Florescu and R. H. Pratt, Eur. Phys. J. D3, 105-111 (1998). 
[28] A. V. Pakhomov, M.S. Thompson and D.A. Gregory, 50- $\mu$ s Time-Delayed Phase Explosion of Elementary Metal Targets, submitted to Journal of Applied Physics. See also http://spacedaily.com/news/laser-02a.htm

[29] D. Javorsek II et al, Phys. Rev. D65, 072003 (2002); D. Javorsek II et al, Phys. Rev. Lett. 87, 231804 (2001); D. Javorsek II et al, Phys. Rev. D64, 012005 (2001). 


\section{Figure Caption}

Figure 1: Possible form of the potential energy of a mirror iron fragment embedded in a piece of lead. 


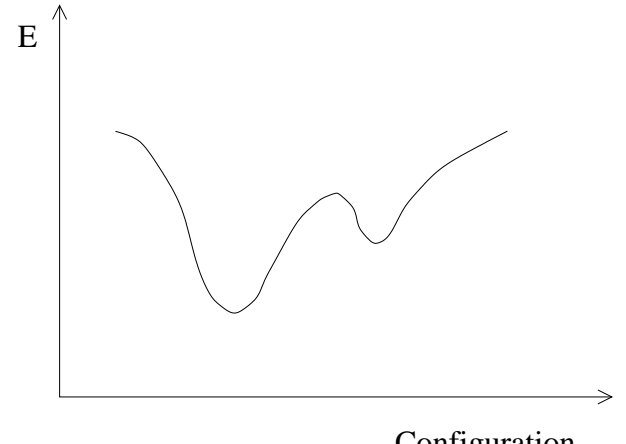

Configuration 